\title{
NGC 7421: Surfing the Intracluster Medium?
}

\author{
Stuart D. Ryder ${ }^{1}$, G. Purcell ${ }^{2}$, D. Davis ${ }^{2}$ and V. Andersen ${ }^{2}$ \\ ${ }^{1}$ School of Physics, University of New South Wales, \\ Sydney, NSW 2052, Australia \\ sdr@newt.phys.unsw.edu.au \\ ${ }^{2}$ Department of Physics \& Astronomy, University of Alabama, \\ Box 870324, Tuscaloosa, AL 35487-0324, USA \\ purcell, davis, andersen@crux.astr.ua.edu \\ Received 1996 August 5, accepted 1996 October 28
}

\begin{abstract}
NGC 7421 is a barred spiral that is displaced from the centre of its optical envelope, and has a 'bow-shock'-like western boundary, both suggestive of interaction with an intracluster medium. We have used five configurations of the ATCA to map the distribution and kinematics of $\mathrm{H}_{\mathrm{I}}$ in NGC 7421, and find supportive evidence for this scenario in the form of an $\mathrm{H}_{\mathrm{I}}$ 'wake'. When compared with ROSAT PSPC observations of the diffuse hot gas in the surrounding group of galaxies, these new ATCA results will allow us to place new constraints on the density and dynamics of the intracluster medium.
\end{abstract}

Keywords: galaxies: individual (NGC 7421) — galaxies: kinematics and dynamics galaxies: intergalactic medium — radio lines: galaxies

\section{Introduction}

Many spiral galaxies display clear asymmetries in their light distributions (Rix \& Zaritsky 1995; Zaritsky \& Rix 1997), including some which are apparently quite isolated and non-interacting. Often, studies of the underlying mass distribution of neutral hydrogen reveal much of this asymmetry to be mainly 'cosmetic', attributable to (in the case of NGC 1313; Ryder et al. 1995) such factors as the presence of an $\mathrm{H}_{\text {I }}$ superbubble. However, the recognition of an inherently non-circular and asymmetric population of galaxies would have major ramifications for topics as fundamental as the derivation of disk inclinations, dark matter halos, and the Tully-Fisher relation.

NGC 7421 is an SB(r)bc galaxy, seen at low inclination, that appears to sit slightly off-centre in a low surface brightness envelope some $2^{\prime}$ in diameter (Figure 1). NGC 7421 exhibits a complete inner resonance ring (Buta 1995), but instead of a complete outer ring, there is a semi-circular arc of $\mathrm{H}$ II regions forming a sharp western boundary to the galaxy. Since NGC 7421 is a prominent member of Group No. 40 in the Catalog of Southern Groups of Galaxies (Maia, da Costa \& Latham 1989), one gets a strong impression that this arc may represent some sort of 'bow-shock' effect as NGC 7421 ploughs its way through the intracluster medium, while the off-centre envelope may represent the 'wake' of its passage. Such bow-shock morphologies are also observed in NGC 2276 (Gruendl et al. 1993) and in NGC 4273 (Davis et al. 1995), both of which are members of groups containing moderate amounts of hot intra-group gas (though probably not enough to explain the presence of the bow-shock).

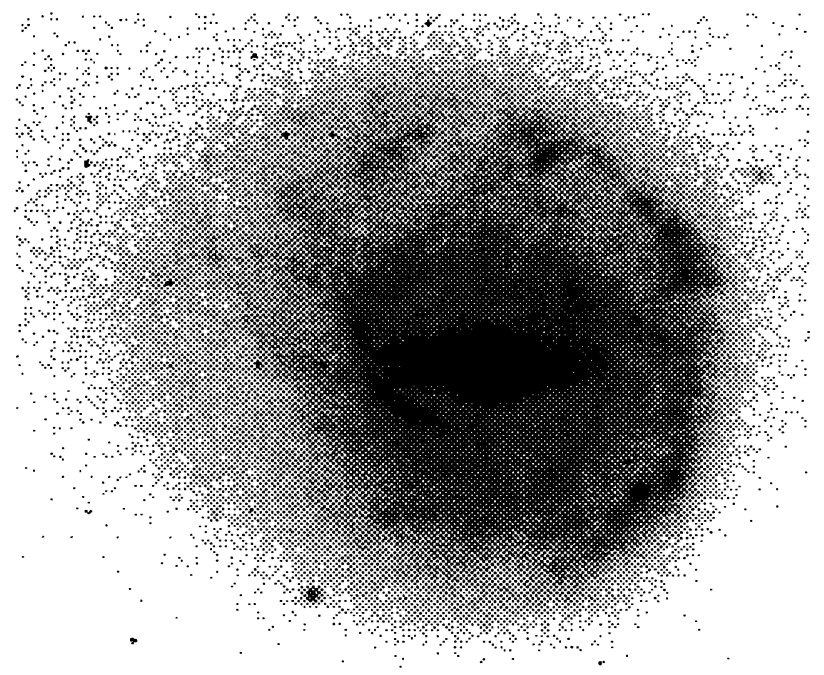

Figure 1-B-band CCD image of NGC 7421 taken with the MSSSO $1.0 \mathrm{~m}$ telescope. North is up, and east is to the left. Note the 'bow-shock' morphology of the western side, compared with the extended, low surface brightness envelope $\left(\mu_{B} \sim 23.7 \mathrm{mag} \operatorname{arcsec}^{-2}\right)$ to the east.

In order to test the hypothesis that NGC 7421 is interacting with an intracluster medium, we are currently analysing some 16000 seconds of publicly available ROSAT Position Sensitive Proportional Counter (PSPC) observations to check whether the density of diffuse hot gas in this group is sufficient to account for any gas sweeping. The dynamics 


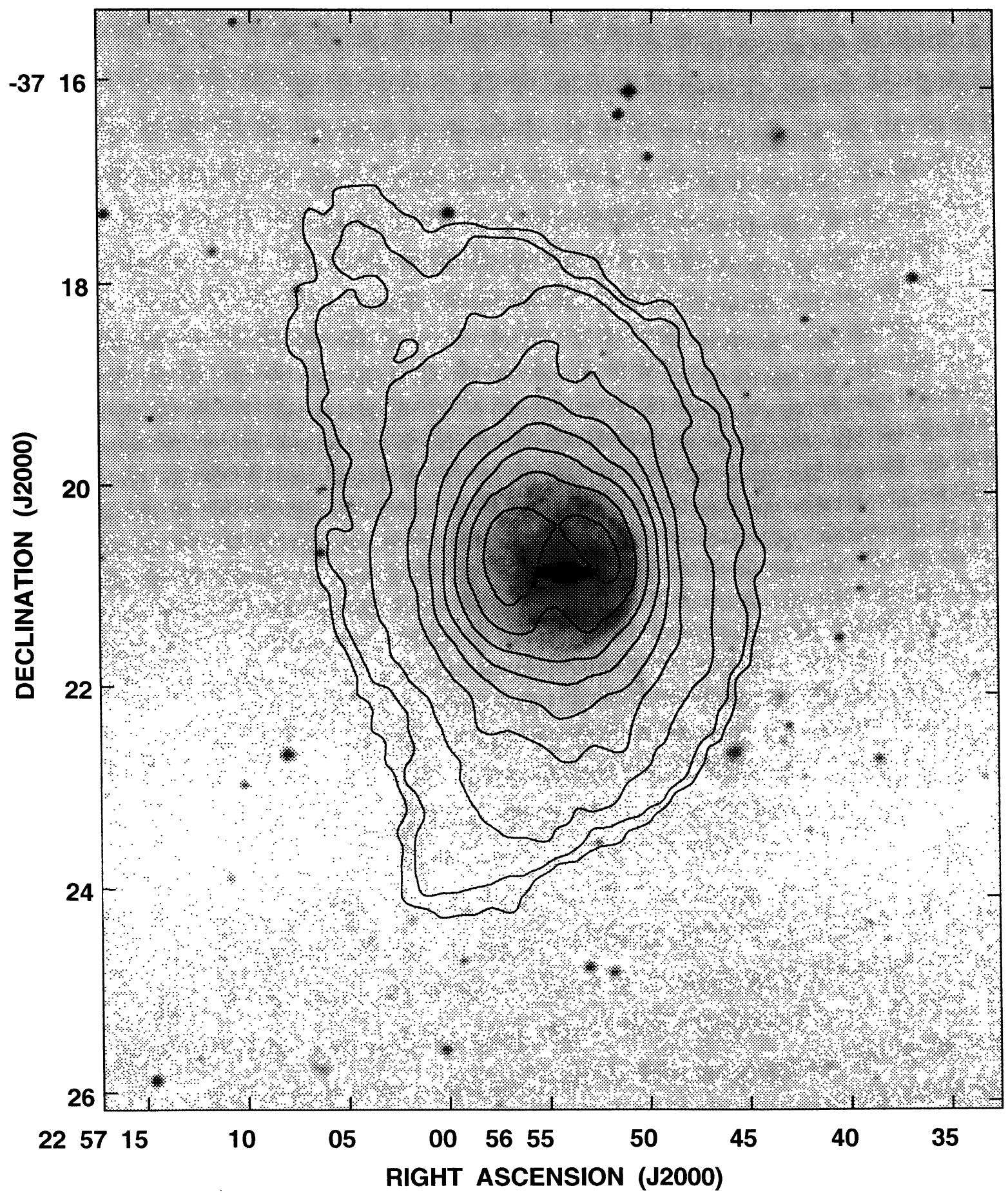

Figure 2-Contours of H I column density (from five configurations of the ATCA) overlaid on a B image of NGC 7421 (from the Digitised Sky Survey). The contours correspond to (projected) column densities of $0 \cdot 2,0 \cdot 4,0 \cdot 9,1 \cdot 8,2 \cdot 6,3 \cdot 5,4 \cdot 4,5 \cdot 3$ and $6.2 \times 10^{20} \mathrm{~cm}^{-2}$. 


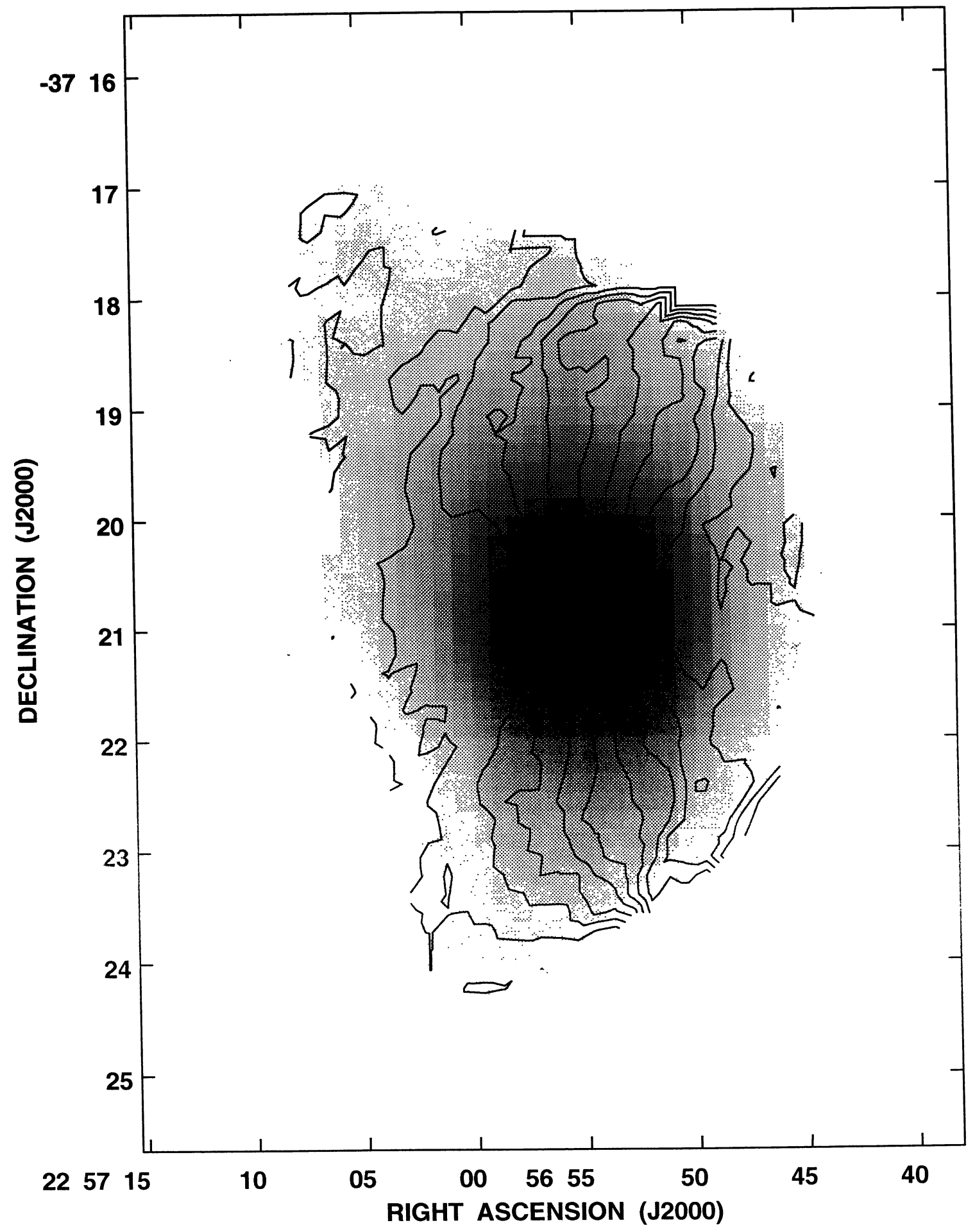

Figure 3-Velocity field of NGC 7421 overlaid on a grey-scale of the $\mathrm{H}$ I column density. The velocity contours span the range from $1730 \mathrm{~km} \mathrm{~s}^{-1}$ (north-east) to $1840 \mathrm{~km} \mathrm{~s}^{-1}$ (western edge of disk).

(C) Astronomical Society of Australia - Provided by the NASA Astrophysics Data System 
of the ionised gas in the inner disk of NGC 7421 have been looked at with the Rutgers Fabry-Perot spectrometer on the CTIO $4 \mathrm{~m}$ telescope (Purcell \& Buta 1997). We have also mapped the distribution and kinematics of the $\mathrm{H}_{\mathrm{I}}$ gas in NGC7421 using a total of five configurations $(0 \cdot 375,750 \mathrm{C}, 1 \cdot 5 \mathrm{D}, 6 \mathrm{C}$ and 6D) of the Australia Telescope Compact Array (ATCA) between 1995 September and 1996 January, and we present here some preliminary results from these observations.

\section{ATCA Results}

Figure 2 shows contours of the $\mathrm{HI}$ column density (resolution $\sim 50^{\prime \prime}$ ) overlaid on an optical image of NGC 7421 from the Digitised Sky Survey ${ }^{1}$. The gas disk extends $\sim 7^{\prime}$ north-south (36 kpc at a distance of $17.8 \mathrm{Mpc}$; Tully 1988), over three times the size of the optical envelope. The impression of a 'bow-shock' from the optical disk is reinforced by the shape of the $\mathrm{HI}$ disk. The northern and southern cusps are consistent with the galaxy ploughing (or rather 'surfing', since the gas has not been entirely stripped) its way westwards through some intracluster medium. There is not significantly more gas underlying the western arc of $\mathrm{H}$ II regions than in the eastern half, though vigorous star formation on the western side may have already consumed much of the accumulated gas.

Figure 3 shows iso-velocity contours from the velocity field overlaid on a grey-scale of the $\mathrm{H}_{\mathrm{I}}$ column density shown in Figure 2. At first glance, the inner velocity field looks fairly normal for a rotating galaxy disk, but there are two highly unusual aspects to it. First, the iso-velocity contours close up on themselves about the kinematic minor axis, but even more intriguing is that the kinematic line-of-nodes (the normal to the iso-velocity contours) and the major axis of the $\mathrm{HI}$ isophotes are perpendicular to each other, instead of the conventional parallel alignment. It is possible that ram pressure has

\footnotetext{
${ }^{1}$ Based on photographic data obtained using the UK Schmidt Telescope. The UK Schmidt Telescope was operated by the Royal Observatory Edinburgh, with funding from the UK Science and Engineering Research Council, until 1988 June, and thereafter by the Anglo-Australian Observatory. Original plate material is (c) the Royal Observatory Edinburgh and the Anglo-Australian Observatory. The plates were processed into the present compressed digital form with their permission.
}

'squeezed' the disk, but then rotation ought to result in elongation of the disk preferentially in one direction. Alternatively, the gas may actually be 'tumbling' about the galaxy's major axis, as in the amorphous dwarf starburst galaxy NGC 5253 (Kobulnicky \& Skillman 1995).

The global $\mathrm{H}$ I profile obtained by integrating the emission within each channel map yields a flux integral of $20 \cdot 2 \mathrm{Jy} \mathrm{km} \mathrm{s}^{-1}$, which equates to a total HI mass of $1.5 \times 10^{9} M_{\odot}$. The profile width at $20 \%$ of the peak flux (uncorrected for inclination) is only $147 \mathrm{~km} \mathrm{~s}^{-1}$. Neutral hydrogen in the 'wake' has been swept to lower velocities, giving rise to a 'single-horn' profile. Such asymmetries in single-dish spectra may be the signature of galaxies akin to NGC 7421 (Richter \& Sancisi 1994).

We were able to successfully extract $\mathrm{H}_{\mathrm{I}}$ data for NGC 7418 (another member of the same group as NGC 7421) from the same observations, even though it lies just outside the $34^{\prime}$ primary beam of the ATCA. The distortion of the gas isophotes in NGC 7418 is consistent with the more usual type of warp, but is further evidence of the turbulent intracluster environment in which NGC7421 and NGC 7418 find themselves.

\section{Acknowledgments}

SDR acknowledges support from EPSCoR grant EHR-9108761, and the receipt of a UNSW ViceChancellor's Postdoctoral Research Fellowship. The Digitised Sky Survey was produced at the Space Telescope Science Institute under US Government grant NAG W-2166.

Buta, R. 1995, ApJS, 96, 39

Davis, D. S., Mushotzky, R. F., Mulchaey, J. S., Worrall, D. M., Birkinshaw, M., \& Burstein, D. 1995, ApJ, 444, 582

Gruendl, R. A., Vogel, S. N., Davis, D. S., \& Mulchaey, J. S. 1993, ApJ, 413, L81

Kobulnicky, H. A., \& Skillman, E. D. 1995, ApJ, 454, L121

Maia, M. A. G., da Costa, L. N., \& Latham, D. W. 1989, ApJS, 69, 809

Purcell, G. B., \& Buta, R. J. 1997, in preparation

Richter, O.-G., \& Sancisi, R. 1994, A\&A, 290, L9

Rix, H.-W., \& Zaritsky, D. 1995, ApJ, 447, 82

Ryder, S. D., Staveley-Smith, L., Malin, D. F., \& Walsh, W. 1995, AJ, 109, 1592

Tully, R. B. 1988, Nearby Galaxies Catalogue (Cambridge Univ. Press)

Zaritsky, D., \& Rix, H.-W. 1997, ApJ, 477, 118 